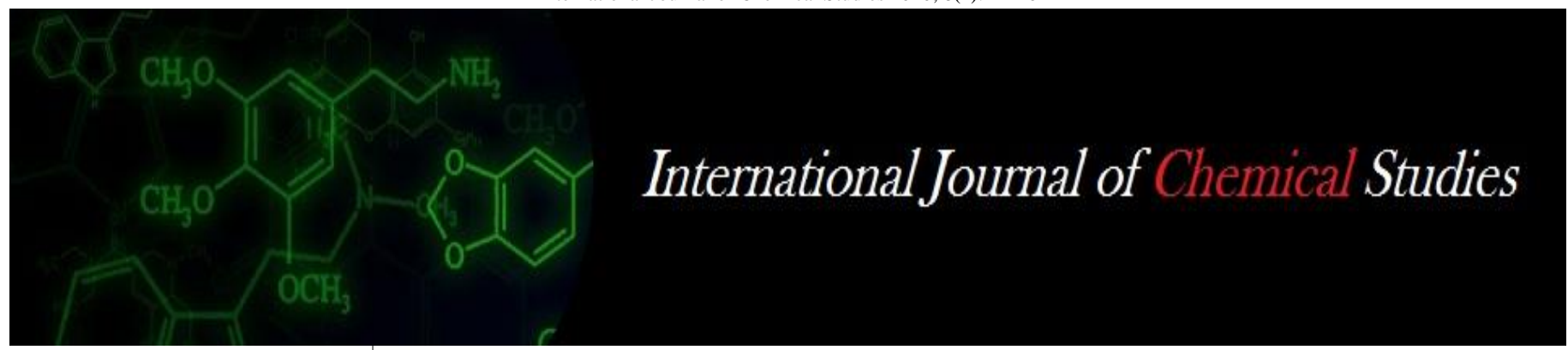

P-ISSN: 2349-8528

E-ISSN: 2321-4902

IJCS 2020; 8(1): 44-46

(C) 2020 IJCS

Received: 24-11-2019

Accepted: 28-12-2019

\section{PR Muthanna}

Department of Plant Pathology,

UAS, Dharwad, Karnataka,

India

\section{MR Ravikumar}

Professor, Department of Plant

Pathology, UAS, Dharwad,

Karnataka, India

\section{DS Sunil Kumar}

Department of Plant Pathology, UAS, Dharwad, Karnataka, India
Corresponding Author:

\section{PR Muthanna}

Department of Plant Pathology,

UAS, Dharwad, Karnataka,

India

\section{Management of tip over disease of banana by using effective PGPRs and different chemicals}

\author{
PR Muthanna, MR Ravikumar and DS Sunil Kumar
}

DOI: https://doi.org/10.22271/chemi.2020.v8.i1a.8343

\begin{abstract}
Banana is one of the most versatile plant that can be used to heal various kinds of illnesses in every aspect. However, its cultivation is hampered by many diseases like in other crops. The least known soft rot disease among farmers caused by Erwinia carotovora subsp. carotovora has became a threat to banana cultivation. The present study was aimed to identify suitable control measures of soft rot to avoid the plunging of banana yield. A field experiment was conducted during 2019 imposing seven different treatments. Among the treatments no disease incidence was recorded streptocycline $(500 \mathrm{ppm})+\mathrm{COC}$ $(3000 \mathrm{ppm})$ and K-cycline $(500 \mathrm{ppm})+\mathrm{COC}(3000 \mathrm{ppm})$. But the influence on plant height $(49.40 \mathrm{~cm})$, pseudostem girth $(20.50 \mathrm{~cm})$, number of leaves per plant (13.08) and number of scorched leaves per plant (0.87) were comparatively less in streptocycline $(500 \mathrm{ppm})+\mathrm{COC}(3000 \mathrm{ppm})$ treatment. Pseudomonas aeruginosa (B-8) + Bacillus cereus (B-13) treatment recorded highest plant height $(52.00 \mathrm{~cm})$, pseudostem girth $(21.57 \mathrm{~cm})$, number of leaves per plant (13.27) and number of scorched leaves per plant (3.17). Pseudomonas aeruginosa (B-8) also significantly influenced the growth promotion followed by Bacillus cereus (B-13). Whereas, untreated control was recorded with highest disease incidence and lowest growth parameters.
\end{abstract}

Keywords: Banana, tip over disease, chemical combinations, plant growth promoting rhizobacteria (PGPR)

\section{Introduction}

Banana is one of the oldest tropical fruit plants known to humanity, and in India which is next to mango. It was regarded as the cheapest, most abundant and most nourishing of all the fruits from time immemorial. Bananas are usually cultivated all year round throughout the nation and grow much better in hot and humid environments. Bananas are the most ideal fruits in the world because they are high in potassium, small in fat and a great source of vitamins and fiber. Bananas and plantains are significant staple food crop in developing nations for millions of individuals. Since, bananas are greater in vitamin $\mathrm{B}_{6}$, anxiety and stress is reduced.

Rhizobacteria are competent bacteria in the rhizosphere capable of multiplying and colonizing on plant roots at all stages of plant growth in the presence of competing microflora (Antoun and Kloepper, 2001) ${ }^{[1]}$ in contact with other microorganisms. Rhizosphere is the soil surface affected by plant root and is considered to play a pivotal role in plant growth and development (Hrynkiewicz and Baum, 2012) ${ }^{[4]}$. Rhizosphere may be neutral, harmful or beneficial to plant growth. Once reintroduced in the rhizosphere, approximately 2 to 5 per cent of rhizobacteria have beneficial effects on plant growth and are referred to as Plant Growth Promoting Rhizobacteria (PGPRs) (Kloepper and Schroth, 1978) ${ }^{[6]}$.

Several factors are attributed to the constraints for high production of banana and the major being the occurrence of diseases. Among the bacterial diseases in Karnataka, tip over or bacterial rhizome banana rot has become important in recent years. The bacterial nature of banana head rot or rhizome rot first noticed in Allahabad (Uttara Pradesh) by Wardlaw (1950) ${ }^{[12]}$. Khan and Nagaraj (1998) ${ }^{[5]}$ from Karnataka recorded the causal organism as Erwinia carotovora. But, Chattopadhyay and Mukherjee (1986) ${ }^{[2]}$ from West Bengal implicated Erwinia chrysanthemi as the causal agent.

\section{Material and Methods}

The experiment on management of soft rot of banana also known as tip over disease of banana was implemented in disease prone field planted with tissue culture banana seedlings. 
The study was conducted during 2019. The experiment comprised of seven treatments with three replications and in each replication 10 banana plants were maintained. The antibacterial chemical combinations and PGPRs were applied to the root zone by scraping the surface soil and treatments were drenched around the pseudostem region. About $1000 \mathrm{ml}$ solution per plant was drenched depending on the plant growth, except bleaching powder, which was applied to the soil at 0.3 per cent per plant at the basin portion of the plant. The chemicals / antibiotics were drenched thrice. The first application was given to 41 days old crop and the second drench was given 20 days later while the third and fourth soil drench was given at 81 and 101 days old plants respectively. The observations on the per cent incidence of disease, plant height, psuedostem girth, total number of leaves and number of leaves scorched were recorded a day before and twenty days after the imposement of treatments.

\section{Results and Discussion}

Field experiment was conducted during 2019 on integrated management of tip over disease of banana caused by $E$. $c$. subsp. carotovora. Treatments were imposed in Randomized Completely Block Design (RCBD). The results (Table 1) indicated that, per cent disease incidence before the initiation of treatments was non-significant among treatments. No disease incidence was recorded in treatments, streptocycline $(500 \mathrm{ppm})+\mathrm{COC}(3000 \mathrm{ppm})$ and $\mathrm{K}$-cycline $(500 \mathrm{ppm})+$ COC (3000 ppm), 20 days after fourth drenching (120 DAP). The next best effective treatment was Pseudomonas aeruginosa (B-8) + Bacillus cereus (B-13) with 13.33 per cent disease incidence followed by Pseudomonas aeruginosa (B-8) (16.67\%) and Bacillus cereus (B-13) (20.00\%) treatments respectively.

The results of present investigation on the efficacy of antibacterial chemical combinations such as streptocycline $(500 \mathrm{ppm})+\mathrm{COC}(3000 \mathrm{ppm})$ and K-cycline $(500 \mathrm{ppm})+$ $\mathrm{COC}$ (3000 ppm) agreed with the findings of earlier workers, wherein it was clearly stated that, agrimycin and streptomycin were very effective in inhibiting the growth of bacteria and in managing the disease under field conditions (Stover, 1959; Mahmoud et al., 1981; Farag et al., 1984; Parashar and Sindhan, 1988 and Nirmaljit Singh et al., 1979) ${ }^{[1,7,3,10]}$.

Among the various antibacterial chemicals and PGPRs applied, highest plant height $(52.00 \mathrm{~cm})$ was recorded in Pseudomonas aeruginosa (B-8) + Bacillus cereus (B-13) followed by Bacillus cereus (B-13) $(50.87 \mathrm{~cm})$, streptocycline $+\operatorname{COC}(49.40 \mathrm{~cm})$ and K-cycline + COC $(49.20 \mathrm{~cm})$ compared to untreated control $(34.58 \mathrm{~cm})$ at 120 days after planting (Table 2). Maximum pseudostem girth $(21.57 \mathrm{~cm})$ was recorded in Pseudomonas aeruginosa (B-8) + Bacillus cereus (B-13) treated plants followed by Bacillus cereus (B13) $(20.78 \mathrm{~cm})$ (Table 3$)$. Maximum number of leaves per plant (Table 4) was recorded in Pseudomonas aeruginosa (B8) + Bacillus cereus (B-13) (13.27) followed by Pseudomonas aeruginosa (B-8) (13.21). Least number of scorched (Table 5) leaves was found in K-cycline + COC (0.92) followed by streptocycline + COC (0.87).

Similarly, Nagaraj (2002) ${ }^{[8]}$ reported that, no disease was recorded in Bacillus subtilis followed by Pseudomonas fluorescence and Vesicular Arbuscular Mychorrhiza (VAM) at 90 days after third application. The maximum plant height $(172 \mathrm{~cm})$ was recorded in mycorrhizal treated plants. Maximum pseudostem girth $(51.61 \mathrm{~cm})$ and number of leaves per plant (29.33) in Bacillus subtilis treated plants.

\section{Conclusion}

Streptocycline $(500 \mathrm{ppm})$ in combination with copper oxychloride (3000 ppm) was found to be the most effective in controlling the pathogen under field condition. Among the PGPRs evaluated, Pseudomonas aeruginosa (B-8) in combination with Bacillus cereus (B-13) was found to be most effective in managing the pathogen under field condition. Pseudomonas aeruginosa (B-8) performed well in managing the disease and also had positive influence on the plant growth.

Table 1: Effect of antibiotics and PGPRs on tip over disease incidence under field condition

\begin{tabular}{|c|c|c|c|c|c|c|}
\hline \multirow{2}{*}{ Treatments } & \multirow{2}{*}{ Concentration } & \multicolumn{5}{|c|}{ Disease incidence (percent disease incidence) } \\
\hline & & $\mid$ Before Drenching (40 DAP) & 60 DAP & 80 DAP & 100 DAP & 120 DAP \\
\hline Pseudomonas aeruginosa (B-8) & $7 \times 10^{6} \mathrm{cfu} \mathrm{ml}^{-1}(1000 \mathrm{ml})$ & $23.33(28.87)^{*}$ & $33.33(35.25)$ & $30.00(33.20)$ & $23.33(28.87)$ & $16.67(24.09)$ \\
\hline Bacillus cereus $(\mathrm{B}-13)$ & $7 \times 10^{6} \mathrm{cfu} \mathrm{ml}^{-1}(1000 \mathrm{ml})$ & $26.67(31.08)$ & $36.67(37.25)$ & $33.33(35.25)$ & $23.33(28.87)$ & $20.00(26.55)$ \\
\hline $\begin{array}{c}\text { Pseudomonas aeruginosa }(\mathrm{B}-8)+ \\
\text { Bacillus cereus }(\mathrm{B}-13)\end{array}$ & $\begin{array}{c}7 \times 10^{6} \mathrm{cfu} \mathrm{ml}^{-1}(500 \mathrm{ml}+500 \\
\mathrm{ml})\end{array}$ & $23.33(28.87)$ & $33.33(35.25)$ & $30.00(33.20)$ & $20.00(26.55)$ & $13.33(21.41)$ \\
\hline Streptocycline + COC & $500 \mathrm{ppm}+3000 \mathrm{ppm}$ & $26.67(31.08)$ & $23.33(28.87)$ & $16.67(24.09)$ & $6.67(14.96)$ & $0.00(0.00)$ \\
\hline K-cycline + COC & $500 \mathrm{ppm}+3000 \mathrm{ppm}$ & $30.00(33.20)$ & $26.67(31.08)$ & $16.67(24.09)$ & $10.00(18.43)$ & $0.00(0.00)$ \\
\hline Bleaching $\mathrm{pc}$ & $3000 \mathrm{ppm}+3000 \mathrm{ppm}$ & $26.67(31.08)$ & $36.67(37.25)$ & $43.33(41.15)$ & $46.67(43.07)$ & $56.67(48.81)$ \\
\hline & - & $23.33(28.87)$ & $36.67(37.25)$ & $43.33(41.15)$ & $53.33(46.89)$ & $63.33(52.71)$ \\
\hline S. & & 2.77 & 2.21 & 2.06 & 2.73 & 1.81 \\
\hline C.D. $(5 \%)$ & & 8.54 & 6.81 & 6.37 & 8.43 & 5.60 \\
\hline
\end{tabular}

*Arc sine transformed value

Table 2: Effect of antibiotics and PGPRs on plant height of banana under field condition

\begin{tabular}{|c|c|c|c|c|c|c|}
\hline \multirow[b]{2}{*}{ Treatments } & \multirow[b]{2}{*}{ Concentration } & \multicolumn{5}{|c|}{ Plant height (cm) } \\
\hline & & $\begin{array}{c}\text { Before Drenching } \\
\text { (40 DAP) }\end{array}$ & 60 DAP & 80 DAP & 100 DAP & 120 DAP \\
\hline Pseudomonas aeruginosa (B-8) & $7 \times 10^{6} \mathrm{cfu} \mathrm{ml}^{-1}(1000 \mathrm{ml})$ & 19.78 & 28.60 & 36.00 & 40.12 & 49.09 \\
\hline Bacillus cereus (B-13) & $7 \times 10^{6} \mathrm{cfu} \mathrm{ml}^{-1}(1000 \mathrm{ml})$ & 21.53 & 28.00 & 38.73 & 44.57 & 50.87 \\
\hline Pseudomonas aeruginosa $(\mathrm{B}-8)+$ Bacillus cereus $(\mathrm{B}-13)$ & $7 \times 10^{6} \mathrm{cfu} \mathrm{ml}^{-1}(500 \mathrm{ml}+500 \mathrm{ml})$ & 18.36 & 28.40 & 39.67 & 45.00 & 52.00 \\
\hline Streptocycline + COC & $500 \mathrm{ppm}+3000 \mathrm{ppm}$ & 21.13 & 29.93 & 37.17 & 43.38 & 49.40 \\
\hline K-cycline + COC & $500 \mathrm{ppm}+3000 \mathrm{ppm}$ & 21.13 & 30.33 & 37.97 & 43.28 & 49.20 \\
\hline Bleaching powder $+\mathrm{ZnSO}_{4}$ & $3000 \mathrm{ppm}+3000 \mathrm{ppm}$ & 20.67 & 25.30 & 29.18 & 34.67 & 37.00 \\
\hline Control & - & 20.33 & 24.67 & 28.67 & 30.83 & 34.58 \\
\hline S.Em. \pm & & 1.14 & 1.18 & 2.44 & 2.47 & 2.61 \\
\hline C.D. $(5 \%)$ & & 3.52 & 3.64 & 7.53 & 7.62 & 8.04 \\
\hline
\end{tabular}


Table 3: Effect of antibiotics and PGPRs on psuedostem girth of banana under field condition

\begin{tabular}{|c|c|c|c|c|c|c|}
\hline \multirow[b]{2}{*}{ Treatments } & \multirow[b]{2}{*}{ Concentration } & \multicolumn{5}{|c|}{ Pseudostem girth $(\mathrm{cm})$} \\
\hline & & \begin{tabular}{|c|} 
Before Drenching \\
(40 DAP)
\end{tabular} & 60 DAP & 80 DAP & 100 DAP & 120 DAP \\
\hline Pseudomonas aeruginosa (B-8) & $7 \times 10^{6} \mathrm{cfu} \mathrm{ml}^{-1}(1000 \mathrm{ml})$ & 5.17 & 6.37 & 13.17 & 16.03 & 20.56 \\
\hline Bacillus cereus (B-13) & $7 \times 10^{6} \mathrm{cfu} \mathrm{ml}^{-1}(1000 \mathrm{ml})$ & 5.00 & 6.50 & 13.17 & 16.03 & 20.78 \\
\hline Pseudomonas aeruginosa (B-8) + Bacillus cereus (B-13) & $7 \times 10^{6} \mathrm{cfu} \mathrm{ml}^{-1}(500 \mathrm{ml}+500 \mathrm{ml})$ & 5.70 & 6.63 & 13.40 & 17.00 & 21.57 \\
\hline Streptocycline + COC & $500 \mathrm{ppm}+3000 \mathrm{ppm}$ & 5.77 & 6.70 & 12.28 & 16.36 & 20.50 \\
\hline K-cycline + COC & $500 \mathrm{ppm}+3000 \mathrm{ppm}$ & 5.00 & 6.70 & 12.27 & 16.33 & 20.32 \\
\hline Bleaching powder $+\mathrm{ZnSO}_{4}$ & $3000 \mathrm{ppm}+3000 \mathrm{ppm}$ & 5.63 & 6.63 & 9.50 & 12.17 & 13.43 \\
\hline Control & & 5.70 & 5.93 & 8.33 & 10.67 & 11.27 \\
\hline S.Em. \pm & & 0.26 & 0.32 & 0.59 & 0.14 & 0.44 \\
\hline C.D. $(5 \%)$ & & 0.73 & 0.99 & 1.8 & 0.42 & 1.34 \\
\hline
\end{tabular}

Table 4: Effect of antibiotics and PGPRs on number of leaves of banana under field condition

\begin{tabular}{|c|c|c|c|c|c|c|}
\hline \multirow[b]{2}{*}{ Treatments } & \multirow[b]{2}{*}{ Concentration } & \multicolumn{5}{|c|}{ Total number of leaves } \\
\hline & & $\begin{array}{c}\text { Before Drenching } \\
\text { (40 DAP) }\end{array}$ & 60 DAP & 80 DAP & 100 DAP & 120 DAP \\
\hline Pseudomonas aeruginosa (B-8) & $7 \times 10^{6} \mathrm{cfu} \mathrm{ml}^{-1}(1000 \mathrm{ml})$ & 8.10 & 8.90 & 11.40 & 12.37 & 13.21 \\
\hline Bacillus cereus (B-13) & $7 \times 10^{6} \mathrm{cfu} \mathrm{ml}^{-1}(1000 \mathrm{ml})$ & 7.99 & 8.63 & 11.00 & 11.80 & 13.17 \\
\hline Pseudomonas aeruginosa $(\mathrm{B}-8)+$ Bacillus cereus $(\mathrm{B}-13)$ & $7 \times 10^{6} \mathrm{cfu} \mathrm{ml}^{-1}(500 \mathrm{ml}+500 \mathrm{ml})$ & 7.30 & 8.87 & 11.67 & 12.50 & 13.27 \\
\hline Streptocycline + COC & $500 \mathrm{ppm}+3000 \mathrm{ppm}$ & 7.83 & 8.80 & 11.00 & 11.67 & 13.08 \\
\hline K-Cycline + COC & $500 \mathrm{ppm}+3000 \mathrm{ppm}$ & 8.00 & 9.03 & 10.77 & 11.73 & 12.21 \\
\hline Bleaching powder $+\mathrm{ZnSO}_{4}$ & $3000 \mathrm{ppm}+3000 \mathrm{ppm}$ & 8.43 & 8.97 & 9.37 & 9.85 & 11.35 \\
\hline Control & - & 8.07 & 8.40 & 8.53 & 8.87 & 9.50 \\
\hline S.Em. \pm & & 0.46 & 0.44 & 0.38 & 0.44 & 0.46 \\
\hline C.D. $(5 \%)$ & & 1.43 & 1.36 & 1.17 & 1.36 & 1.41 \\
\hline
\end{tabular}

Table 5: Effect of antibiotics and PGPRs on number of scorched leaves under field condition

\begin{tabular}{|c|c|c|c|c|c|c|}
\hline \multirow{2}{*}{ Treatments } & \multirow{2}{*}{ Concentration } & \multicolumn{5}{|c|}{ Number of scorched leaves } \\
\hline & & Before Drenching (40 DAP) & 60 DAP & 80 DAP & 100 DAP & 120 DAP \\
\hline Pseudomonas aeruginosa (B-8) & $7 \times 10^{6} \mathrm{cfu} \mathrm{ml}^{-1}(1000 \mathrm{ml})$ & 1.60 & 2.55 & 3.87 & 3.95 & 3.40 \\
\hline Bacillus cereus (B-13) & $7 \times 10^{6} \mathrm{cfu} \mathrm{ml}^{-1}(1000 \mathrm{ml})$ & 1.70 & 2.97 & 4.01 & 3.92 & 3.42 \\
\hline $\begin{array}{c}\text { Pseudomonas aeruginosa }(\mathrm{B}-8)+\text { Bacillus } \\
\text { cereus }(\mathrm{B}-13)\end{array}$ & $7 \times 10^{6} \mathrm{cfu} \mathrm{ml}^{-1}(500 \mathrm{ml}+500 \mathrm{ml})$ & 1.10 & 2.22 & 3.63 & 3.92 & 3.17 \\
\hline Streptocycline + COC & $500 \mathrm{ppm}+3000 \mathrm{ppm}$ & 1.00 & 1.28 & 1.30 & 1.17 & 0.87 \\
\hline K-cycline + COC & $500 \mathrm{ppm}+3000 \mathrm{ppm}$ & 1.13 & 1.87 & 1.90 & 1.26 & 0.92 \\
\hline Bleaching powder $+\mathrm{ZnSO}_{4}$ & $3000 \mathrm{ppm}+3000 \mathrm{ppm}$ & 2.10 & 2.44 & 4.70 & 5.07 & 5.30 \\
\hline Control & - & 1.27 & 3.01 & 5.13 & 6.07 & 6.31 \\
\hline S.Em. \pm & & 0.24 & 0.26 & 0.1 & 0.23 & 0.18 \\
\hline C.D. $(5 \%)$ & & 0.74 & 0.79 & 0.31 & 0.72 & 0.56 \\
\hline
\end{tabular}

\section{References}

1. Antoun H, Kloepper JW. Plant growth-promoting rhizobacteria (PGPRS), In: Encyclopedia of Genetics. Ed. Brenner, S. and Miller, J. H., Academic Press, New York, 2001, 1477-1480.

2. Chattopadhyay PK, Mukherjee NA. Pseudostem rot of banana due to Erwinia chrysanthemi pv. paradisiaca. Curr. Sci. 1986; 55:789-790.

3. Farag NS, Lashin SM, Abdel-All RS, Shatiz HM, SeifElyazal AM. Antibiotica and control of potato black leg and brown rot diseases. Agric. Res. Rev. 1984; 60:149166.

4. Hrynkiewicz K, Baum C. The potential of rhizosphere microorganisms to promote the plant growth in disturbed soils In: Environmental protection strategies for sustainable development, strategies for sustainability. Ed: Malik A. Grohmann E, Springer, London, New York, 2012, 35-64.

5. Khan ANA, Nagaraj MS. Occurrence of a new bacterial disease on banana in Karnataka, In: Annu. Meet. Symp. Integrated Disease Management and Crop Loss Assessment, Indian Phytopath. Soc. (Southern Chapter), UAS, Bangalore, December 10-12, 1998, 73.

6. Kloepper JW, Schroth MN. Plant growth promoting rhizobacteria on radishes. In: Proc. $4^{\text {th }}$ Int. Conf. Plant
Pathogenic Bacteria. Ed. Station de Pathologic Vegetal et Phytobacteriologic. Angers, France. 1978; 2:879-882.

7. Mahmoud SAZ, Ishac YZ, Abdel-Hafez AM, Farag NS, Fawzi FG. Senstivity of soft rot bacteria to antibiotics and the role of ampicillin- resistant mutants in rot development. Res. Bull., Faculty of Agriculture, Ain Shams Univ, Cario, Egypt. 1595, 1981, 16.

8. Nagaraj MS. Etiology, ecology and management of tip over disease of banana caused by Erwinia carotovora subsp. carotovora (Jones) Holland. M. Sc. (Agri) Thesis, Univ. Agric. Sci., Bangalore, Karnataka, 2002, 30-103.

9. Nirmalajit Singh BS, Thind, Harnam Singh. Control of bacterial rot of potato tubers. Indian Phytopath. 1979; 32:645-646.

10. Parashar RD, Sindhan GS. Efficacy of Ktorocin and other chemicals in controlling soft rot of potato in field and storage. Indian J Mycol. Plant Pathol. 1988; 18:39-42.

11. Stover RH. Bacterial rhizome rot of bananas. Phytopathology. 1959; 49:290-292.

12. Wardlaw CW. Banana diseases VIII, Notes on the various diseases occurring Trinidad. Trop. Agric. 1950; 11:143-149. 